\title{
AN ANALYSIS OF CONTENT AND COMMUNICATION PATTERNS OF THAI ELDERLY FAN PAGES
}

\author{
Panarat Lim* \\ Dhurakij Pundit University, Thailand
}

\begin{abstract}
Since 2005, Thailand has been moving fast to become an aging society. The number of Thai citizens aged 60 or older has steadily risen. The government, as a result, has come up with policiies to supports the elderly to gain knowledge and access to news and media. According to communication technology development, social media is one of many forms of technology that is growing steadily. A new trend of older people using Facebook to communicate, learn, and share has increased. The researcher aim to learn about the content and communication patterns on elderly Facebook fan pages. Findings would be used to enhance the quality of those Facebook fan pages. The objectives of this research are (1) to identify the varieties of contents on the fan pages generated by Pages admin (Manager or Content Creator) and (2) to analyse communication patterns available on those elderly Facebook Fan Pages. Purposive sampling was used to collect the data. To analyse the content four Facebook fan pages ---namely @Oldbutnotchicken, @thaigri, @elderenergy, and $@$ GenOldThaiPBS were used for data collection. The research findings from the content analysis show that the Pages Administrators generated contents which could be classified into six areas namely, (1) health, (2) exercise, (3) psychology and mental health, (4) activities, (5) learning a skill, and (6) elderly laws. From the socialization analysis, it was found that there are intellectual, spiritual and behavioural contents. Furthermore, communication patterns of all the fan pages relied on verbal communication as such infographics, videos, YouTube, and live videos to share content on the page as well. However, it was found that there was a lack of engagement from the audience. Research results could be used as guidelines for improving the content and communication patterns on the Elder Facebook fan pages as an effective learning source for senior citizens lifelong learning.
\end{abstract}

Keywords: Elderly, Facebook, Fan Pages, Socialization, Communication, Online Media

\section{Introduction}

In 2017, approximately 11.23 million Thai were considered the elderly. The figure represents 17.13 percent of the total population. In 2021, Thailand is expected to be a elderly society completely. Like The Nation, Thai Portal mentions the following:

"Thailand will become a full-fledged aging society in 2021 when their number will rise to 13.1 million or 20 per cent of the total population," according to NESDB, a National Economic and Social Development Council Board report. 


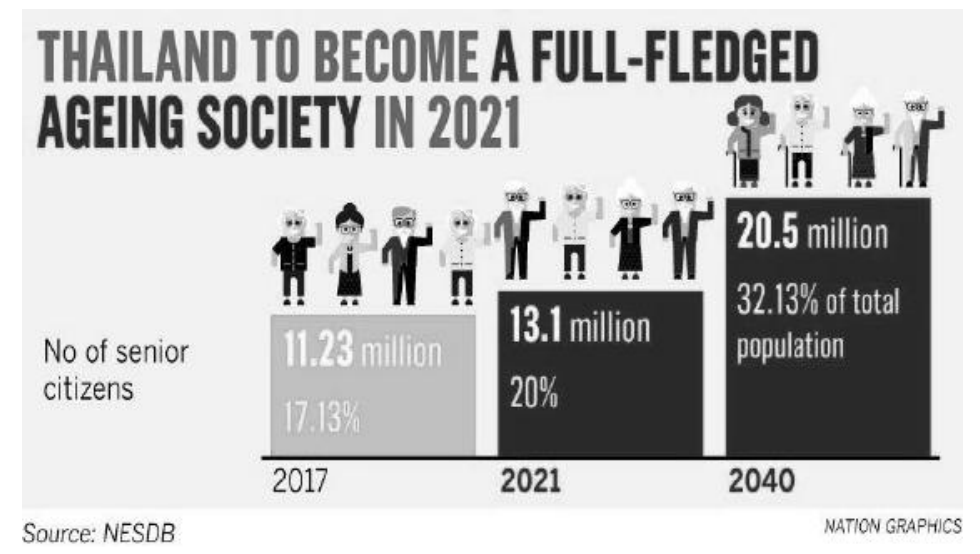

Figure 1 Thailand will become a full-fledged aging society in 2021 from "Risks grow for aging population" (2017, December 17) by Wichit Chaitrong.

http://www.nationmultimedia.com/detail/Economy/30333636. December 11, 2017

For readiness preparation of the people for their quality aging, the Thai government has prepared Thai society for the aging society, as seen from the strategy in the National Economic and Social Development Plan and the National Elderly Plan No. 2 (2002-2021). There are six strategies for the promotion and development of the elderly, one of them is the strategy on processing, upgrading and disseminating knowledge on the elderly and the national monitoring of the implementation of National Plan on the Elderly (NPE), the measure to support the elderly to gain knowledge and have access to news and media. So they are able to learn from information such as Health Information and share experience among people, and take part as the social development resources and enable to live with dignity and sustain their healthiness and living standard.

The $2^{\text {nd }}$ National Plan on the Elderly (2002-2021)

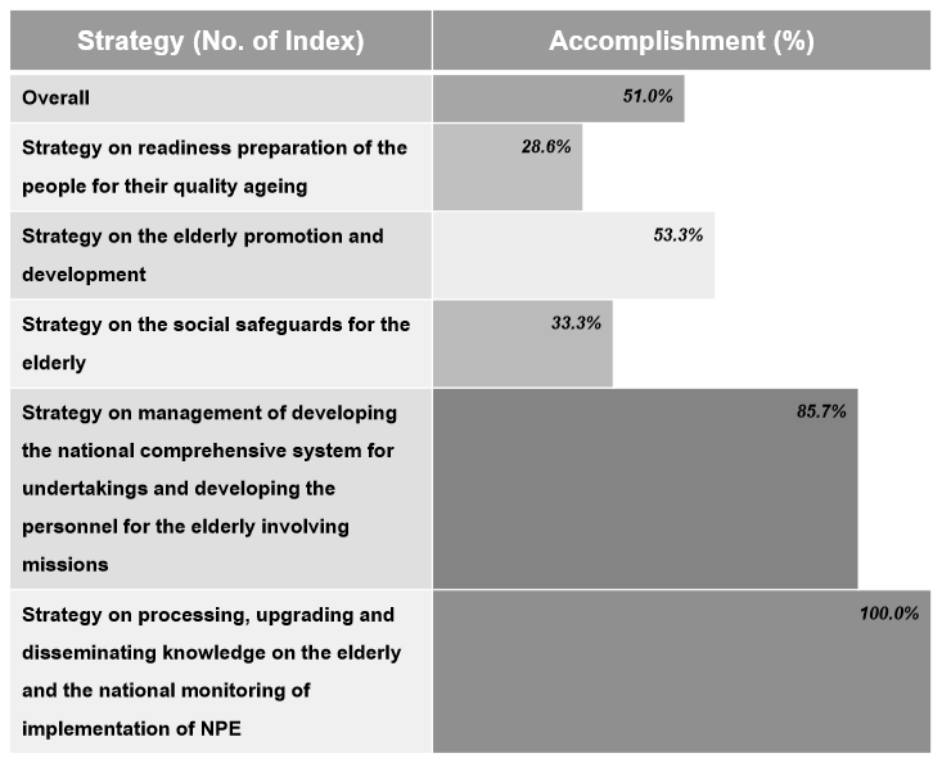

Figure 2 The 2nd National Plan on the Elderly (2002-2021) from "Public Symposium on Aging in East and Southeast Asia in Seoul on February 28" by Suwanrada W. (December 11, 2017) https://www.toyotafound.or.jp/english/international/2016/toyotafound/data/Worawet_Suwanrada.pdf.

Due to the rapid change of information technology, many communication channels to occur that helps people communicate more easily, whether are a mobile phone, smartphone and internet, especially social networks 
become an indispensable thing today. Changing in communication technology, new innovations and social media such as Facebook Line, Instagram, etc. are affecting everyone's lifestyle including the elderly. The number of Facebook users in Thailand from 2017 to 2023. In 2019, is expected to reach 26.9 million, up from 25.7 million in 2017 (https://www.statista.com/statistics/490467/number-of-thailand-facebook-users/). Meanwhile, Thonzocial data analysis services provider in Thailand disclosure of information about the use of Facebook media of Thai elderly in 2017 increased by 25\% from 2016 (Thoth Zocial. https://www.posttoday.com/it/475356(. Facebook popular among Thai elderly is used to communicate, to learn, to share for reducing feelings of loneliness and increase the mental well-being of older adults.

From document study, it found that elderly people are seeking health information. The elderly's motivation for seeking health information are health problem, disease prevention, and treatment of diseases and nutrition, including the elderly need for health information format such as voice, text, images and multimedia (Porchita Upatum, 2016). In addition, it was found that The elderly people have 3 aspects of using Facebook (1) In terms of usage, such as viewing news on Facebook of children, finding information they like and writing comments (2) In the chat room, for example, communicate with acquaintances or close friends and Family (3) The use of benefits, such as tracking news faster, sharing knowledge and experience, spending time usefully and reducing loneliness.

Therefore, the researcher curious to find out what kind of content does the elderly fan pages have and how the communication patterns are presented. The results could be used as guide- lines to develop and enhance the quality of the elderly Facebook fan pages that support elderly need.

The purpose of the study can be summarized as follows:

1. To identify the varieties of content on Elderly Fan Pages generated by Pages admin

2. To analyse the communication patterns on those Elderly Facebook fan pages.

\section{Literature review}

\section{Theories of Socialization}

Socialization is the means by which human infants begin to acquire the skills necessary to perform as a functioning member of their society and is the most influential learning process one can experience. There are different Forms of Socialization as such Group Socialization is the theory that an individual's peer groups, rather than parental figures, influences his or her personality and behavior in adulthood. Gender socialization refers to the learning of behavior and attitudes considered appropriate for given sex and Cultural socialization refers to parenting practices that teach children about their racial history or heritage. (Lumen Boundless Sociology, Online).

Socialization is a social learning process consits of attention, retention reproduction and motivation. (Bundura, 1977)

Attention Processes. This process is determined by both the observers' characteristics and the arrangement of intended behviors.

Retention Processes. The learning process that observers who code modeled activities into either words, concise labels, or vivid imagery learn and retain behavior better than those who simply observe or are mentally preoccupied with other matters while watching 
Motor Reproduction Processes. This process due to individuals can execute accurate demonstration of a new behavior through modeling. Then, they refine the action through self-corrective based on feedback and focus demostrations of behavioral segments that have only been learned in part.

Motivational Processes. Motivation is inspired by percieved rewards or purnishments. Many factors influence one's ability to observed and then engage in a given behavior. Also Bundura (1977) note that not every person not correctly reproduce every behavior.

\section{Social Learning Process}

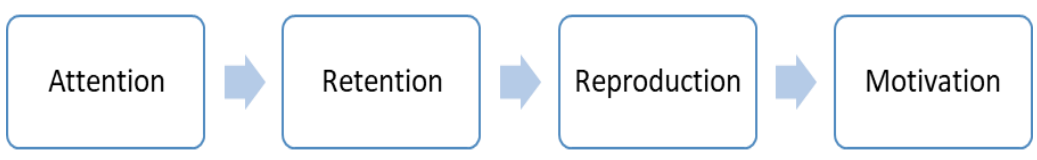

Figure 3 Social learning process from "Applying Communication Theory for Professional: A Practical Introduction” by Dainton $M$ and Zelley E.D. London: SAGE Publications, 2005.

Mass media influences us by providing knowledge and understanding, creating knowledge, skills, building strength and creating values for the audience. (Kaewthep, 2004). Agents of socialization include a group of people who are responsible to refine members to have the knowledge, ability, skills, attitude, ideology, etc. in the way that society needs. Important social representatives include family, peer group, school, reference groups which are anticipatory socialization and retrospective socialization. Moreover, Daniel Levinson's theory of positive adult development and how it influenced changes in the perception of development during adulthood. Positive adult developmental processes are divided into at least six areas of study: hierarchical complexity, knowledge, experience, expertise, wisdom, and spirituality (Lumen Boundless Sociology, Online). Bandura's (1977) social learning theory claim as following:

"most human behavior is learned observationally through modeling: from observing others one forms an idea of how new behaviors are performed, and on later occasions this coded information serves as a guide for action." (Dainton, M \& Zelley E.D., 2005, p.206)

\section{Facebook}

Social media applications, make it easier for elders to contact and share information with family and friends through text, voice and images. (Mayela Coto, Fulvio Lizano, Sonia Mora, and Jennifer Fuentes, 2017). Facebook is a form of communication capable of reaching people at all levels that affect people lifestyle and to interact, share experiences, knowledge. Moreover, exchange stories and share interests between groups of people. These groups are called facebook fan pages which is the public area for creating various activities that encourage people in the group to have a conversation, exchange ideas. Unlike the personal Facebook profile, fan pages are visible to everybody on the internet. Anyone on Facebook can connect to and receive updates from a page by becoming a fan. (George, 2012) Moreover, Facebook fan pages provide information continuously, realtime interaction and content creation among users and communities including creating a dialogue between the Page Administrators and the target group more quickly. Facebook fan page Plan must be considered in order to create an attractive page image on the Facebook fan page and creates an impression to the audience. Moreover, Facebook fan pages as a two-way communication medium that allow the Page Administrators and the elderly audiences to deliver the information, share opinions and knowledge between each other for self-development. Two-way Communication by discussing with the group, posting interesting and relevant content to the target group. Post update question, create new topics for discussion, post pictures or videos and Tag fan pages, which will help to spread widely )Sakkawatra, 2011(. Thus, it is the opportunity to gain knowledge reducing dependency on others. 


\section{Communication Patterns}

Communication patterns are classified by communication channel (1) One-way Communication is the communication that the senders sent the message to the receivers without feedback from the receivers. (2) Twoway Communication is the communication between the senders and the receivers interact and feedback to each other. )Vali Khan Thurin, Piyapak Sin Buathong, 2003). Moreover, communication patterns are classified by symbol (1) Verbal Communication refers to the use of sounds and language to relay a message. Verbal Communication requires either a speaker (or writer) to transmit the message, and a listener (or reader) to make sense of the message. Interpersonal is one of four types of verbal communication. Interpersonal is form of communication between two individual and two individual will swep their role sender and receiver in order to communicate clearer manner. (MFLF, http://www.maefahluang.org/?p=17) There are different communication form interpersonal, group and mass and media tools such as YouTube, clipped video, and infographic. (2) Nonverbal Communication occur without using any oral or written word. It relies on physical movement, color, signs, symbol, express feeling, attitude or information. Types of non-verbal communication are facial expression, posture and body orientation, body language, space and distance, para-linguistic, and visual communication. ect. (iEduNote, https://iedunote.com/nonverbal-communication)

\section{Data collection and sample}

This research methodology was a qualitative method using purposive sampling to target elderly Facebook fan pages. The aim was to investigate data collection-content and communication patterns. This study investigated the contents posted by those Elderly Fan Page during the period of 1 July to 31 December 2018, using the keyword "SungVai (elderly)" with a combination of the following keyword: "non-profit organisation, communities" on Facebook fan pages. The search returned the list of 10 elderly fan pages. Four Facebook fan pages were selected as the sample with the most followers on fan page with high number more than 1,000 followers --- namely (1) Old people, not chickens “@oldbutnotchicken” With 10,190 followers, (2) Elderly "@ thaigri" has 9,800 followers, (3) Elderly power "@elderenergy" has 7,200 followers, and (4) Elderly together "@GenOldThaiPBS" has 3,400 followers. For the research purposes, the researcher considered types of contents that were posted on elderly Facebook fan pages and communication patterns which created by the Page Administrators. Using coding sheet as a tool for contents analysis, the researcher created a categorized table, classifying types of content and communication patterns that appear on Elderly Facebook fan pages.

\section{Results}

Results indicated that Facebook Fan Page @ Oldbutnotchicken is the most popular Elderly Facebook Fan Pages. The page entails knowledge of health care, healthy food, an exercise that are summarized into infographic fomats. Most content types are Inspiration by sharing elderly experience and the various of activities. Page Administrators was guest speaker to share knowledge in the elderly seminar through the movie activities "The Bucket List" organized by the Younghappy organization and the Office of the Health Promotion Foundation (SSI). The Communication Patterns of the @ Oldbutnochicken fan page mostly is link from the other websites. Moreover, Page Administrators creates her own content using the video clips and infographic to communicate with verbal communication to the audience. 


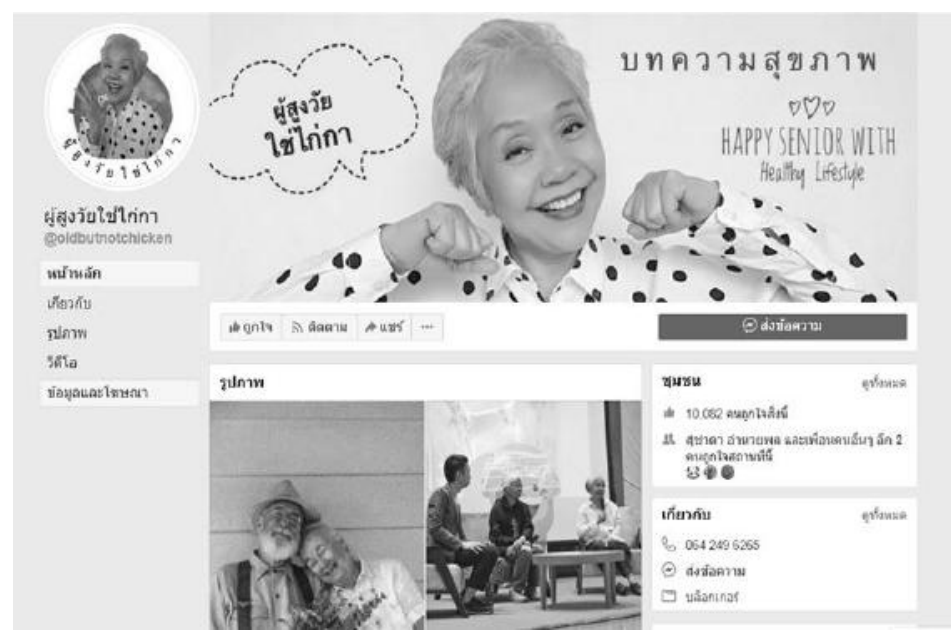

Figure 4 Page Administrators @ Oldbutnochicken news shared knowledge in elderly seminar

Facebook fan page called @ thaigri is created by the Thai Elderly Research and Development Institute (PSU). They are posted behavioral content regarding social norm and social role, knowledge content about elderly protection right, elderly news, knowledge-driven dialogue, elderly rights protection system. Property management of the elderly, health news on exercise and healthy food for the elderly. The communication patterns by sharing news links from other website, such as policy of Elderly Protection Right from government organisation mostly, the elderly health news, health education training, and inspiration content respectively. Moreover, using verbal communication via video clips and infographic to share elderly lifestyle stories.

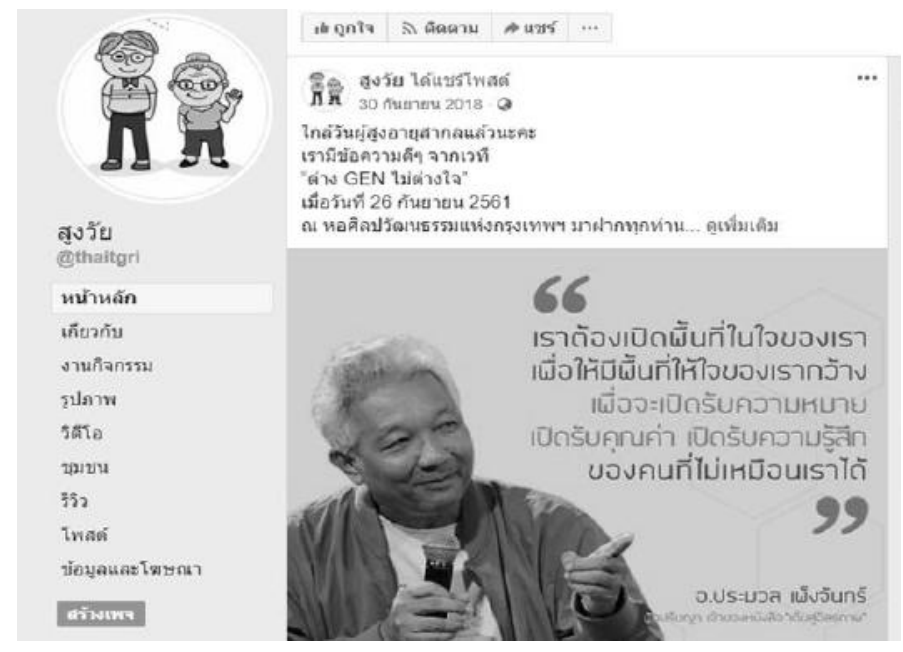

Figure 5 Inspiration content for elderly @ thaigri Fan Page

Facebook fan page called "@elderenergy" focuses on encouragement of the old people to live a happier live by learning from the other elderly activities such as triathlon exercise, stories telling for encouraging the elderly happy living and suggesting about activities for the elderly. Knowledge about health care and nutrition information for promoting self-development of the elderly. Most of the contents were of the real stories of various people to encouraging and inspiring the old people. Communication patterns are interpersonal communication to sharing news links from other websites, blogs, clipped videos, and infographics. 


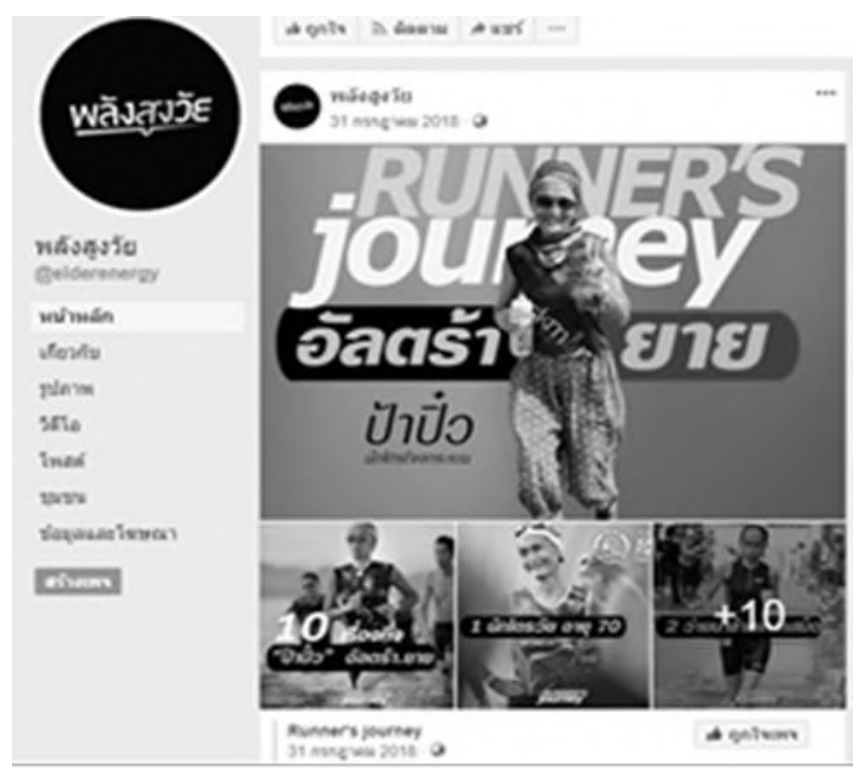

Figure 6 Encourage old people to exercise for health.

Facebook fan page called “@GenOldThaiPBS”)Elderly Together( is created by Thai PBS (Television station). The content types focus on technology knowledge, seminar and public dialogue, public relations for inviting the audience to join the GenO (LD) documentary program about the elderly society. Most of content poste from other websites toward the GenOldThai fan page. Communication patterns used link from blog, YouTube, Facebook live for encouragement and inspiring the elderly audience.

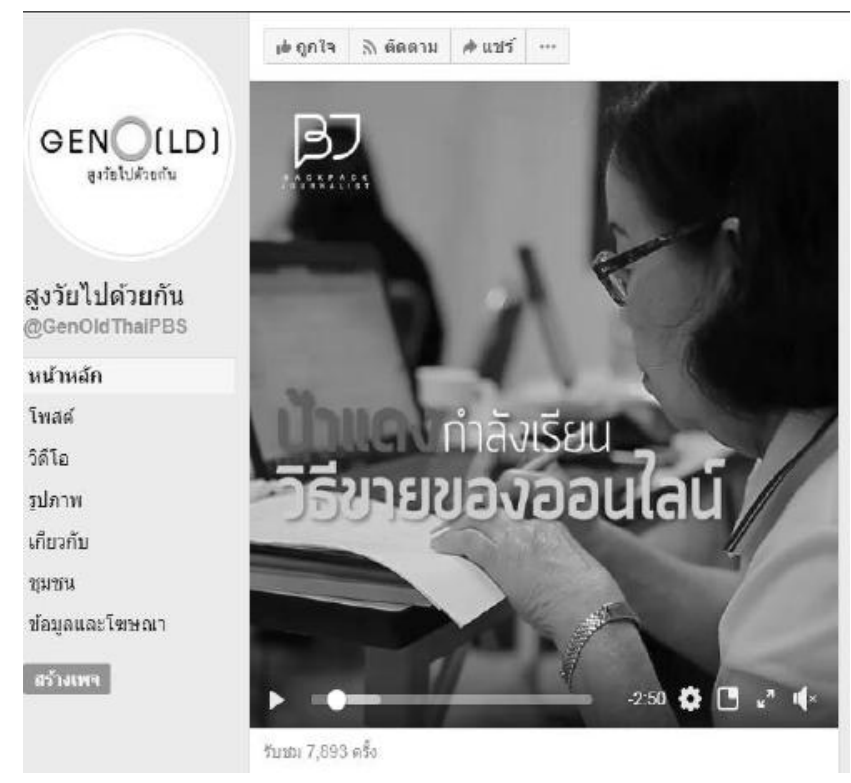

Figure 7 Inspire elderly to learn how to sell product online

\section{Discussions}

In social psychology, it is recognized that social learning can occur through the media. )Bundura, 1971(. Albert Bandura explained that most of the learning due to the observation of the model or imitation from the model only one model can convey the whole idea and expressions simultaneously. Based on the research results, there are page contents that promote socialization processes in various aspects. The varieties of contents 
available on all 4 elderly pages can be divided into 3 dimensions, including (1) intellectual socialization content, (2) spiritual content and (3) behavioral content. The intellectual content deals with knowledge and social skills including health, exercise, seminar, law, career, saving and properties. Spiritual content which are inspiration, encouragement, entails ethic, social value, encouragement, lifestyle, and the behavior dimension covers activities in social norm and social role.

The model that is presented through the Facebook fan pages media will motivate elderly audience develop better skills and providing the skills and habits necessary for participating within his or her own society including learns the attitudes, values and actions appropriate to individuals (Theories and practices in social psychology, Online). The model in the content of knowledge on the elderly Facebook fan pages such as health care and nutrition information, elderly protection right, inspiration contents that will affect the audience in their self-development because of Social Learning/Socialization Theory Expansion of media types to various new media types. Media can create a long-term impact on learning. Bundura's social learning theory note that human behavior is learned observationally through modeling: from observing others one forms an idea of how new behaviors are performed, and on later occasions this coded information serves as a guide for action." (Dainton, M \& Zelley E.D., 2005, p.206). The elderly would create balance in their life; they will reduce stress and give them other outlets to express themselves and have fun.

Facebook Media changes the way of new communication such as poking in Facebook, wall post, photo display an invitation to joy activities with Facebook, up to date content and create a way to express feelings that are effective in maintaining relationships. Social practices were shared information about their lives with others, frequency of sharing, the person who will communicate with which has an impact on sociology, our behaviour and relationships with individuals, organizations and brands. Facebook seems to be a safe place to share messages, and what will help to increase the response from friends in the group is trust. Referring to the feeling of trust in passing information among friends that are shared (Shih, 2011). Fan Pages has the ability to foster community engagement through two-way communication and connect to and receive updates from a page. (David, 2012).

New media is communication technology, such as audio, text, graphics or video/animation that has the potential to link with network technology includes computers and/or telecommunications that causing many changes in communication such as Facebook, email, etc. (Siriyuwasak and others 2004). The communication patterns of elderly Facebook fan page are interpersonal communication. Elderly Page Administrators send the messages about the elderly information --health news, technology knowledge, inspiration content, elderly protection right, training activities-- to the senior audience. Using verbal communication such as written content, clipped videos, and info graphic. This would be able to motivate the audience. Especially, video clips with animation and sound can attract the audience attention more than others.

However, the lack of audience engagement was found to be an issue. Facebook fan page plan must be considered in order to create an attractive page image on the Facebook fan page and creates an impression to the audience )Sakkawatra, 2011(. Social learning process should apply to create Facebook fan page. Page Administrators have to determine the characteristics and need of the elderly for providing content to build their attention, and to provide varieties of content and activities for elderly retention. Moreover, in reproduction process, the content could be use as a model for elderly to refine the action through self-corrective. and motivate the audience to participate by sharing their experience and knowledge. According to adult developmental processes that are divided into hierarchical complexity, knowledge, experience, expertise, wisdom, and spirituality (Lumen Boundless Sociology, Online). So, improving the content should be done making it reliable, up to dated, events to be update too and provide knowledge, experience, spirituality contents to share with the elderly on Facebook fan page. Two-way Communication by discussing with the group, posting interesting and relevant content to the target group )Sakkawatra, 2011(. They can provide feedback to elderly fan pages. Media features such as viewpoint and motion can be configured in ways to create "fields of potential action" that engage viewers and optimize conditions for learning. (Salah L., Robb L., Shuai W., and Roy D.P., 2018). 


\section{Conclusions}

In conclusion, this article analyzed the content and communication patterns of the Facebook fan pages for the elderly. The findings indicates that all the Page was the dissemination of elderly events, news, and useful information, emphasis most of the intellectual socialization content regarding health, exercise, a seminar on the elderly. Furthermore, most of the spiritual contents include inspiration, encouragement and most of the behaviour content regarding activities organised by various organisations. Adult development has to be a concern for selecting the content and design the communication patterns. Only one of four elderly fan pages, @ oldbutnotchicken, created her own content and produced the video clips to communicate with the audience. The communication patterns of all 4 elderly fan pages were that most of them were using verbal communication in the form of a message, photo, and sound to disseminate the content. To provide the fan page more interesting and more followers, the Page Administrators must focus on two-way communication with the audience. They should write their own content by choosing those that the elderly want to know. Moreover, active discussion and attracting quick attention invite effective engagement. Page Administrators should be aware of what the varieties of content related to audience needs and the two-way communication patterns that will be given, basic beliefs and, social values. They should make sure that pages are full of learning and skills that will give the elderly people iinspiration, ideas, and attitudes, necessary for social life to survive and be able to live a happy life and to encourage the elderly to learn and prepare themselves for self-development.

\section{References}

Dainton, M. and Zelley, E. D. (2005). Applying Communication Theory for Professional Life: A practical Introduction. London: SAGE Publications.

George, A. D. (2012, August 19). What is a Fan Page on Facebook? You Need to Know This!. Retrieved from https://blog.heyo.com/what-is-a-fan-page/.

iEduNote. Non-verbal communication: Difinition, Types, Importance (Explained). Retrieved form https://iedunote.com/nonverbal-communication

Kaewthep, K. (2004). Media Analysis: Concepts and Techniques. Bangkok, TH: Higher Press.

Mayela Coto, Fulvio Lizano, Sonia Mora, and Jennifer Fuentes. Social Media and Elderly People: Research Trends. Retrieved from Springer International Publishing AG 2017 G. Meiselwitz (Ed.): SCSM 2017, Part II, LNCS 10283, pp. 65-81, 2017. DOI: 10.1007/978-3-319-58562-8_6.

MFLF. Four typs of verbal communication. Retrieved from http://www.maefahluang.org/?p=17)

Pornchita Upatum. (2016(. Health Information Seeking Behavior of Elderly. Palichart Journal Thaksin University, 29(2), 71-87.

Rattanavarata, P. and Pimchangthong, D. (2018). Influence of Media Characteristics and Media Access on Information Exposure Via Digital Signage: A Case Study of Medium-Level Fresh Coffee Shops. Suthiparithat, 32(103), 148-159.

Salah L., Robb L., Shuai W., and Roy D.P. (2018, March 16). Learning With Media Harnessing Viewpoint and Motion to Generate Fields of Potential Action. Journal of Media Psychology. Retrieved from https://doi.org/10.1027/1864-1105/a000235.

Sakkawatra, S. (2011). Facebook Marketing, edited by Vilas Chamlertwat. iMarketing 10.0. Bangkok, TH: Provision). 52-60.Theory of Socialization. Retrieved from https://courses.lumenlearning.com/boundlesssociology/chapter/theories-of-socialization/.

Shih, C. (2011). The Facebook Era. Bangkok, TH: Praphan San.

Siriyuwasak, U. and others. (2004). Introduction to journalism: Media, Culture and Society. Bangkok, TH: Chulalongkorn University Press.

Suwanrada, W. (2017, February 28). Public Symposium in Seoul, Korea Sharing Responses on the Ground Aging Society and the Surrounding Challenges in Asia. Retrieved from https://www.toyotafound.or.jp/english/international/2016/toyotafound/data/Worawet_Suwanrada.pdf. 
Theories and practices in social psychology. $\quad$ Retrieved from http://elearning.psru.ac.th/courses/270/\%E0\%B8\%9A\%E0\%B8\%97\%E0\%B8\%97\%E0\%B8\%B5\%E0\%B9\%88 \%202.pdf.

Thoth Zocial. The Thai population on Facebook aged 18-24 years is the highest increase. (2018, January 12). Retrieved from https://www.posttoday.com/it/475356.

Wichit Chaitrong. (2017, December 17). Risks grow for aging population. Retrieved from http://www.nationmultimedia.com/detail/Economy./30333636.

Vali Khan Thurin, Piyapak Sin Buathong. (2003). An analysis of communication culture in organization: a case study of humanities and social sciences faculty Khon Kaen University. Humanities, Social Sciences (Khon Kaen University), 21(4), 1-14. 